SLAC-PUB-11228

May, 2005

\title{
KLYSTRON LINEARIZER FOR USE WITH 1.2 MW 476 MHZ KLYSTRONS IN PEP-II RF SYSTEMS*
}

\author{
J. Fox**, T. Mastorides, D. Teytelman, D. Van Winkle, Y. Zhou \\ Stanford Linear Accelerator Center, Menlo Park, CA 94025, U.S.A \\ A. Gallo, LNF-INFN, Frascati, Italy
}

\begin{abstract}
The direct and comb loop feedback around the RF cavities in PEP-II is critical in reducing longitudinal instabilities driven by the cavity impedance. The non-linear 1.2 MW klystron is in the signal path for these feedback loops. As a result, the effective small-signal gain of the klystron at $85 \%$ saturation reduces the impedance control by factors of 5 to 20 as compared to a linear power amplifier. A klystron linearizer circuit has been developed which operates in series with the power amplifier and acts to equalize the small and large signal gains through the combination. The technique must implement a $1 \mathrm{MHz}$ linear control bandwidth over roughly $15 \mathrm{~dB}$ of RF signal level variation. The dynamics of this system is operating point dependent, and the channel must have dynamic gain compensation to keep the linearity compensation loop stable over changes in operating point. The design of this non-linear signal processing channel (incorporating RF and DSP techniques) and measured results from full-power klystron testing are presented.
\end{abstract}

Presented at the 2005 U.S. Particle Accelerator Conference

Knoxville, Tennessee, USA

May 16 - May 20, 2005

* Work supported by U.S. Department of Energy contract DE-AC02-76SF00515. 


\title{
KLYSTRON LINEARIZER FOR USE WITH 1.2 MW 476 MHZ KLYSTRONS IN PEP-II RF SYSTEMS*
}

\author{
J. Fox**, T. Mastorides, D. Teytelman, D. Van Winkle, Y. Zhou \\ Stanford Linear Accelerator Center, Menlo Park, CA 94025, U.S.A
}

A. Gallo, LNF-INFN, Frascati, Italy

\section{Abstract}

The direct and comb loop feedback around the RF cavities in PEP-II is critical in reducing longitudinal instabilities driven by the cavity impedance. The nonlinear 1.2 MW klystron is in the signal path for these feedback loops. As a result, the effective small-signal gain of the klystron at $85 \%$ saturation reduces the impedance control by factors of 5 to 20 as compared to a linear power amplifier. A klystron linearizer circuit has been developed which operates in series with the power amplifier and acts to equalize the small and large signal gains through the combination. The technique must implement a $1 \mathrm{MHz}$ linear control bandwidth over roughly $15 \mathrm{~dB}$ of RF signal level variation. The dynamics of this system is operating point dependent, and the channel must have dynamic gain compensation to keep the linearity compensation loop stable over changes in operating point. The design of this non-linear signal processing channel (incorporating RF and DSP techniques) and measured results from full-power klystron testing are presented.
\end{abstract}

\section{INTRODUCTION}

The low level RF systems in PEP-II use direct and comb loop feedback techniques to reduce the driving impedance from the cavity fundamental impedance [1]. These techniques use the 1.2 MW high power klystron as a signal element in a feedback path. The high power klystron has a saturating input/output characteristic as the drive power is increased towards maximum output power at a given high voltage power supply voltage. As a result, the large signal gain of the klystron (Pout/Pin) is significantly different from the small signal gain (dPout/dPin) - for a typical operating point the small signal gain is reduced by factors of 5 to 20 . The direct/comb loop impedance control effectiveness is reduced due to this saturation effect (the cavity impedance is reduced by $1 /$ small signal gain), so that instability growth rates due to the cavity impedance can be much greater than can be readily controlled via the longitudinal and low group delay woofer instability control systems $[2,3]$.

We have developed a klystron linearizer function which acts in series with the klystron, so that the series product of the two has a constant (linear) small signal gain over variations in klystron output level. The heart of this

\footnotetext{
* Work supported by U.S. Department of Energy contract DE-AC0276SF00515

**jdfox@slac.stanford.edu
}

technique is an amplitude sensing feedback network, sampling the klystron input and klystron output, and adjusting an RF drive level modulator to keep the ratio of the two signals through the klystron path a constant. Such an amplitude linearizing loop is not a new idea [4], and in recent years related linearizer techniques have become important for communications amplifiers[5]. The application of this technique to the LLRF systems and instability control was initially suggested by Alessandro Gallo. Our implementation (Figure 1) only corrects the klystron amplitude or magnitude non-linearity - any phase nonlinearity vs. operating level is not corrected with this technique. However in our application is it amplitude modulation which must be transferred through the klystron by the direct/comb loops to suppress phase modulation of the beam[6,1], and so this simpler amplitude only control loop was developed.

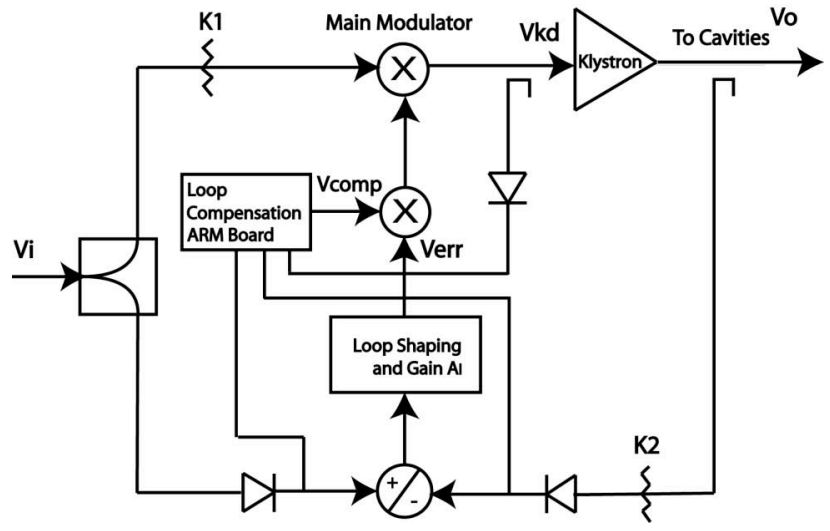

Figure 1: Loop implementation.

\section{IMPLEMENTATION}

Table 1 lists the important specifications for the circuit.

Table 1: Linearizer specifications

\begin{tabular}{|l|l|}
\hline Center frequency & $476 \mathrm{MHz}$ \\
\hline Amplitude linearity correction range & $15 \mathrm{~dB}$ \\
\hline Closed loop bandwidth & $1 \mathrm{MHz}$ \\
\hline Compensation loop bandwidth & $10 \mathrm{~Hz}$ \\
\hline Noise Floor -additive below carrier & $<-40 \mathrm{~dB}$ \\
\hline
\end{tabular}

The circuits are implemented in a mix of RF and baseband electronics. Figure 1 shows the drive path to the high power klystron, with a wideband RF modulator (Analog Devices 834) as the gain controlling element. A fraction of the LLRF $V_{i}$ signal is coupled to a diode detector to measure forward power, similar diodes are 
used with a directional coupler and attenuators in the high power klystron drive and output. The Vi and klystron output signals are processed via baseband amplifiers, and a high gain summing node and error amplifier are used to generate the error signal controlling the RF modulator.

The basic topology is straightforward; however there are several important design considerations. As the action of the linearizer is to boost the level of modulation signals, the linearizer operating point must be carefully matched against the klystron saturation curve - if the modulation levels drive the klystron to saturation (the inflection point in the power out curve) the sign of the linearizer loop gain inverts, and the positive feedback pushes the loop "over the top", causing the power output of the klystron to decrease. Starting the RF station, with LLRF signals but no klystron HV, causes this condition and the linearizer must have special circuits and software to recognize this situation. Another issue is the loop gain of the feedback path is a function of the system input level. Figure 2 shows the variation in closed loop bandwidth vs. +/- $1 \mathrm{~dB}$ variation in input level. For the required $13 \mathrm{~dB}$ operating range the linearizer loop gain must be compensated to avoid peaking or loop instability. Referring to figure 1, the loop gain is:

$$
T_{L}=\frac{V_{i}^{2}}{V_{k d}} V_{c o m p} K_{1} A_{l}
$$

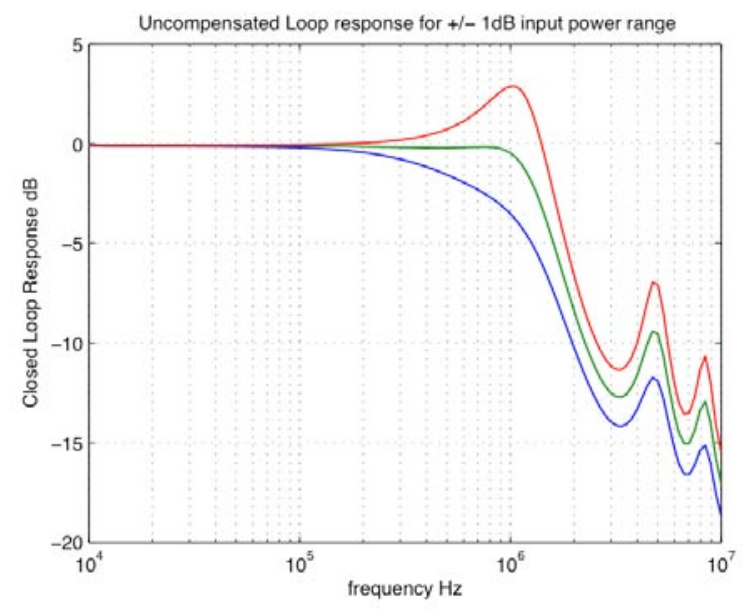

Figure 2: loop bandwidth vs. input level.

The closed loop response of the linearizer loop must be well-damped, and it is important to have nearly $1 \mathrm{MHz}$ bandwidth in the loop to achieve good impedance control over the full direct loop bandwidth. To compensate the loop gain variation, a second wideband modulator in the feedback control loop adjusts the loop gain at different system input levels, as shown in figure 1. The compensation voltage is set to be (where $k_{c}$ is a scaling constant, and $\mathrm{V}_{\mathrm{kd}}$ is the klystron drive level):

$$
V_{\text {comp }}=\frac{k_{c} V_{k d}}{V_{i}^{2}}
$$

Our initial implementations used an analog technique to implement the $1 / \mathrm{x}^{2}$ function - the linearizer prototype uses a digital microcontroller (ADUC7020), which measures the input drive level and klystron drive levels via the diode detectors, and digitally computes a correction voltage to be applied to the modulator controlling the feedback loop gain.This digital process samples at $1 \mathrm{KHz}$ rate and allows the loop gain to track the slow variations in klystron operating point. The digital controller coefficients and operating software allow flexibility in choosing compensation loop bandwidth. The digital controller software also allows the development of monitoring and fault recording functions to study the linearizer in operation.

The linearizer is designed to correct the compression of the klystron small signal gain, and it must do so over the operating range of the high power klystron. While the power required from the klystron varies with beam current over a $100 \mathrm{~kW}$ to $1.2 \mathrm{MW}$ range, the actual operating point variation of the klystron drive signal is relatively small, as the design of the LLRF regulating loops adjust the klystron high voltage supply to keep the klystron input drive level at a regulated operating point. With this input level regulated, the linearizer then only must correct for the small signal gain variation due to saturation effects, which determines the $13-15 \mathrm{~dB}$ dynamic range of the linearizer.

These initial linearizers are being developed to demonstrate the feasibility of the technique and to understand via beam measurements the necessary linearizer performance. As it is not possible to easily quantify the contribution of the linearizer if only 1 of four accelerator klystrons is linearized, we constructed 5 complete prototype linearizers, with the intent to operate 4 in the PEP-II LER (with 1 for high power klystron test stand operation). These prototype units have complete RF functionality, though the external remote control interface is minimal, and configuration of the linearizer largely determined via front panel hardware settings. The front panel allows adjustment of the operating points (reference and feedback path gains) and a signal loop tool feature allows the in-situ measurement of the linearizer loop gain necessary to adjust the $1 / \mathrm{x}^{2}$ compensation. An external monitor module is used to bring out internal signals as well as send logic signals indicating incorrect operating points of the internal loops and limiter functions.

\section{LINEARIZER TESTING}

Testing such a non-linear circuit function requires some special care. Initial testing and development of the linearizer technique used a klystron model circuit (a low power non-linear circuit which mimicked the RF saturation and amplitude non-linearity of the high power system). High power tests were also performed on a klystron test stand, where the full power behavior of the linearizer and klystron combination could be exercised. 


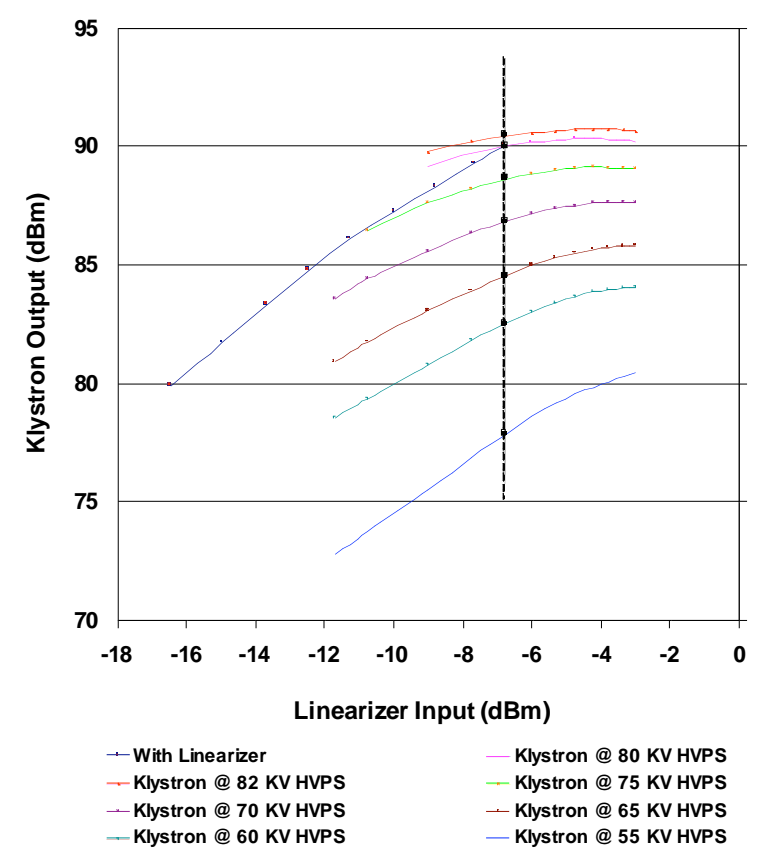

Figure 3: Klystron power output vs. linearizer input (DC power sweep) showing linearized small signal gain as well as saturated klystron transfer functions. $90 \mathrm{dBm}$ is 1 MW.

Figure 3 shows a full-power amplitude sweep of the linearizer-klystron combination on the test stand with the family of unmodified klystron input-output characteristics for the HV supply range of $55-82 \mathrm{KV}$. The klystron drive level is held constant at $44 \mathrm{dBm}$ over the power sweep via the HV supply regulation. The linearizer action is clearly seen in the linearized klystron output, as is the compression of the klystron, especially at the high output powers.

It is also important to study the dynamic behavior of the linearizer. One useful test is to study the response to an amplitude modulated carrier. The carrier to sideband ratio should remain constant in a linear amplifier. Observing the linearizer output (the compensated klystron signal from the linearizer) shows the increased levels of the sidebands, (as well as harmonics of the modulation) which are then compressed in the klystron.

Table 2: Carrier/Sideband Ratios at different modulation depths for $476 \mathrm{MHz}$ carrier, $10 \mathrm{KHz}$ modulation.

\begin{tabular}{|l|l|l|}
\hline Carrier/Sideband Ratios & $2.6 \% \mathrm{AM}$ & $10 \% \mathrm{AM}$ \\
\hline Input Signal & $-37.7 \mathrm{~dB}$ & $-25.9 \mathrm{~dB}$ \\
\hline $\begin{array}{l}\text { Linearizer Output } \\
\text { (to Klystron) }\end{array}$ & $-31.6 \mathrm{~dB}$ & $-20.1 \mathrm{~dB}$ \\
\hline Klystron Output & $-39.0 \mathrm{~dB}$ & $-27.1 \mathrm{~dB}$ \\
\hline $\begin{array}{l}\text { Raw Klystron } \\
\text { (no linearizer) }\end{array}$ & $-44.6 \mathrm{~dB}$ & $-32.2 \mathrm{~dB}$ \\
\hline
\end{tabular}

Table 2 shows the carrier/sideband ratio at various points in the processing chain for $2.6 \%$ and $10 \%$ modulation. The un-linearized output shows compression of the $2.6 \%$ small-signal modulation by $-7 \mathrm{~dB}$, while the linearized klystron output spectrum shows the original carrier/sideband ratio is restored to within $-1.3 \mathrm{~dB}$. This residual error is due to the finite gain of the control loop.

\section{SUMMARY AND FUTURE DIRECTIONS}

At the time of the PAC conference 4 linearizer units are installed in the LER and we are anticipating initial beam testing in late spring 2005. A preliminary test with a single linearizer in summer 2004 showed a measurable growth rate reduction[7], but more careful configuration of the linearizer operating point, and linearization of all klystrons in the LER, is required for quantitative measurements of the growth rates. This information is important in predicting the operation of the LLRF and broadband longitudinal feedback for upgraded operating currents and additional RF cavities[8].We are particularly interested in understanding the depth of small-signal modulation present in the operating machine over variations in current, injection, transient disturbances, etc. which will determine the practical limits of this technique.

\section{ACKNOWLEDGEMENTS}

The authors thank the ARDA and Accelerator departments for funding this initial prototype technology effort.

\section{REFERENCES}

[1] F. Pedersen, "RF Cavity Feedback", B Factories, The State of the Art in Accelerators, Detectors and Physics, SLAC-400, pp. 192 - 207

[2] D. Teytelman, L. Beckman, D. Van Winkle, J. Fox, A. Young (SLAC), "Development and Testing of a Low Group-delay Woofer Channel For PEP-II", EPAC-2004- THPLT155, SLAC-PUB-10559, Jul 2004.

[3] J. Fox, et al, "Programmable DSP based Multi-Bunch Feedback: Operational Experience from Six Installations" Proc. Beam Inst. Workshop, Cambridge MA 2000

[4] F. E. Terman, R. R. Russ, "Some Notes on Linear and Grid-Modulated Radio Frequency Amplifiers", Proc. IRE, 1941, Vol . 29, pp 104-107.

[5] Ultra RF Application Notes , "An Overview of Linear Amplifier Systems", http://www.cree.com/ftp/pub/appnote1.pdf

[6] D. Teytelman, "PEP-II Low-level RF tutorial", presented SLAC 6-7 August 2003

[7] D. Teytelman, "Klystron linearizer test results", PEPII group meeting, 24 June 2004

[8] J. Seeman, et al, "Design for a $10^{36}$ Super-B-Factory at PEP-II", EPAC2004-MOPLT143, SLAC PUB 10548, Jul 2004. 